\title{
Quantum Circuits Assisted by Local Operations and Classical Communication: Transformations and Phases of Matter
}

\author{
Lorenzo Piroli $\oplus^{1,2}$ Georgios Styliaris, ${ }^{1,2}$ and J. Ignacio Cirac ${ }^{1,2}$ \\ ${ }^{1}$ Max-Planck-Institut für Quantenoptik, Hans-Kopfermann-Straße 1, 85748 Garching, Germany \\ ${ }^{2}$ Munich Center for Quantum Science and Technology (MCQST), Schellingstraße 4, D-80799 Mnchen, Germany
}

(Received 14 April 2021; accepted 26 October 2021; published 23 November 2021)

\begin{abstract}
We introduce deterministic state-transformation protocols between many-body quantum states that can be implemented by low-depth quantum circuits followed by local operations and classical communication. We show that this gives rise to a classification of phases in which topologically ordered states or other paradigmatic entangled states become trivial. We also investigate how the set of unitary operations is enhanced by local operations and classical communication in this scenario, allowing one to perform certain large-depth quantum circuits in terms of low-depth ones.
\end{abstract}

DOI: 10.1103/PhysRevLett.127.220503

Recently, we have witnessed the formation of close connections between quantum information theory (QIT) and quantum many-body physics (QMBP). A potential area of common interest among the already established ones is the classification of quantum states and operations. For instance, in QIT one is interested in states that are related by local operations and classical communication (LOCC) since entanglement is seen as a resource and those operations do not increase it [1]. In QMBP, instead, one is interested in the phases of matter that are dictated by local (unitary) transformations [2-6] since those are the ones typically occurring in nature. Despite the apparent similarities, the goals and methods in these fields are very different. First, the notion of locality is not the same. In QIT, there is no underlying geometry, so QIT usually refers to operations that act on a qubit (or subset of qubits) independent of their location. In QMBP, instead, there is an underlying geometry (typically a lattice), and locality refers to operations or Hamiltonians acting on subsystems close to each other. In addition, in QIT measurements and communication are allowed, while these are not traditionally considered in QMBP scenarios (although, recently, much attention has been devoted to unitary dynamics in many-body systems subject to repeated measurements; see, e.g., [7-13]).

The advent of noisy intermediate-scale quantum devices [14] has attracted the interest of the QIT and QMBP communities, providing a unique framework to share

Published by the American Physical Society under the terms of the Creative Commons Attribution 4.0 International license. Further distribution of this work must maintain attribution to the author(s) and the published article's title, journal citation, and DOI. Open access publication funded by the Max Planck Society. methodologies and pursue common goals. Those devices operate quantum circuits (QCs), where quantum gates act on nearest neighbors according to some lattice geometry. Additionally, single qubit measurements can be performed and local gates applied depending on the outcomes. Thus, it is very natural to consider the classification of states, phases of matter, or actions in general under a new paradigm that includes both the local operations appearing in QMBP and the LOCC of QIT. This has clearly practical motivations: for instance, accounting for LOCC could improve the efficiency of recent preparation protocols of topologically ordered states with quantum devices [15], possibly scaling to a larger number of qubits. At the fundamental level, this problem provides a common ground for QIT and QMBP, with the potential to motivate a fruitful cross fertilization of ideas.

In this work, we establish a framework to address this question. We consider state transformations and unitary operations via finite-depth QCs assisted by LOCC, highlighting how this leads to new possibilities. We show that paradigmatic examples, such as the toric-code (TC) [16], the Greenberger-Horne-Zeilinger (GHZ), and $W$ states $[17,18]$, appear in the trivial phase. Furthermore, we provide a full classification of phases in 1D in the context of matrix product states (MPS) [19-21], extending that analyzed in $[2,22]$. For operations, LOCC enhances the potential of QCs, enabling the implementation of unitary transformations that would require complex QCs, which may become useful in the design of future quantum computers.

Quantum circuits and LOCC.-We consider spins arranged over an $N \times \cdots N=: \Lambda_{N, D}$ regular lattice in $D$ spatial dimensions. The associated Hilbert space is $H=H_{d}^{\otimes M}$, with $M=N^{D}$ spins. The local space is $H_{d}$, has dimension $d$, and we will call $\{|0\rangle, \ldots,|d-1\rangle\}$ the computational basis. We denote by $\mathcal{U}$ the set of unitary 
transformations acting on the spins. We begin by introducing the class of QCs as the operators $V \in \mathcal{U}$ that are decomposed as a sequence of unitaries $V=V_{\ell} \ldots V_{2} V_{1}$, where each "layer" $V_{n}$ contains quantum gates acting on disjoint pairs of nearest-neighbor spins [23]. We call $\ell$ the circuit depth.

Definition 1 (depth- $\ell$ quantum circuits): $\mathcal{Q C}_{\ell} \subset \mathcal{U}$ is the set of unitaries that can be expressed as quantum circuits of depth $\ell$.

In the context of QIT, it is often useful to extend certain operations to include extra resources [24]. Here, we consider adding ancillas (initialized in a product state) of identical Hilbert space $H_{d}$ to each lattice site. We then introduce the set of local unitaries (LU), denoted by $\mathcal{L U}$, as follows: $U \in \mathcal{L U} \subset \mathcal{U}$ if $U=\bigotimes_{i=1}^{M} u_{i}$, where $u_{i}$ acts only on the $i$ th local spin and its associated ancillas. We will consider ancillas and local unitaries as "free resources," i.e., we will be allowed to add as many ancillas as needed and perform arbitrarily many local unitary operations.

When ancillas are available, we may modify the action of $V \in \mathcal{Q C}_{\ell}$ by adding local operations between single layers of unitaries, that is, $V^{\prime}=U_{\ell} V_{\ell} \ldots U_{2} V_{2} U_{1} V_{1} U_{0}$, where $U_{n} \in \mathcal{L U}$. Note that, in general, $V^{\prime}$ is not a unitary operator on $H$ since $U_{n}$ also acts on the ancillas. Finally, we will consider an additional extension of the allowed operations, including LOCC: after a QC (which may include additional ancillas), we allow for local (orthogonal) measurements on the ancillas, and LU depending on the outcomes of the measurements, which are classically communicated among all the qudits. Classical communication will always be considered a "free" operation.

State transformations with QCs and LOCC.-The addition of measurements gives rise to randomness. Thus, by adding LOCC to QCs, it might seem difficult to extend the class of states that can be prepared deterministically, but it is indeed possible. This is not surprising since in the context of QIT there are several instances where measurements, if followed by LOCC, can lead to deterministic transformations [25]; see, e.g., [26-28].

We address the question: when can a product state $|\mathbf{0}\rangle=$ $|0\rangle^{\otimes M} \in H$ be (deterministically) transformed into another one, $|\varphi\rangle \in H$, using only QCs or QCs together with LOCC? For the first case, there exists $U \in \mathcal{Q C}_{\ell}$ such that $|\varphi\rangle=U|\mathbf{0}\rangle$. For the latter, we restrict ourselves to the following scheme. We first apply a depth- $\ell$ circuit, with possibly local unitaries acting in between different layers of gates, as explained previously. Then, we sequentially measure each ancilla $a_{i}$ in some orthonormal basis, $\left\{\left|\varphi_{k_{i}}\right\rangle_{i}\right\}$ and apply $U \in \mathcal{L U}$ depending on the outcomes of all previous measurements (so, overall, we perform up to $M$ measurements and apply $M \mathrm{LU})$. Note that in this protocol we perform a single measurement per site. One could also define a more general scheme with multiple rounds of LOCC [29]. While this would not change our conclusions, we restrict ourselves to the above definition.
Definition 2 (transformations under QCs and LOCC): We say that a state $|\varphi\rangle$ can be prepared by $X=\mathrm{QC}_{\ell}, \mathrm{QCcc}_{\ell}$ if it can be obtained, respectively, by $U \in \mathcal{Q C}_{\ell}$ or $U \in \mathcal{Q C}_{\ell}$ together with LOCC, using the above procedures. We will write $|\mathbf{0}\rangle \stackrel{X}{\rightarrow}|\varphi\rangle$.

Let us analyze the power of LOCC. For that we give a simple necessary condition for transformations using QCs. In the following, we define the distance between two regions $A, B \subset \Lambda$ as $d(A, B)=\min _{i \in A, j \in B} d(i, j)$, where we denote by $d(i, j)$ the minimal number of edges connecting the vertices $i$ and $j$ in the graph associated with the lattice $\Lambda$.

Proposition 1: Let $A, B \subset \Lambda$ with $d(A, B)>2 \ell$ and $X_{A}, Y_{B}$ operators supported on $A$ and $B$, respectively. If $|\mathbf{0}\rangle \stackrel{\mathrm{QC}_{\ell}}{\longrightarrow}|\varphi\rangle$, then

$$
\left\langle\varphi\left|X_{A} Y_{B}\right| \varphi\right\rangle=\left\langle\varphi\left|X_{A}\right| \varphi\right\rangle\left\langle\varphi\left|Y_{B}\right| \varphi\right\rangle .
$$

See [30] for a proof. This proposition is useful to prove that some states cannot be prepared by QCs, as we now exemplify.

Example 1 (the GHZ and W states): Let us consider qubits in a 1D lattice $(M=N)$ with periodic boundary conditions (PBC). The GHZ and $\mathrm{W}$ states are $[17,18]$

$$
|\mathrm{GHZ}\rangle=\frac{1}{\sqrt{2}}\left(|0\rangle^{\otimes N}+|1\rangle^{\otimes N}\right), \quad|W\rangle=\frac{1}{\sqrt{N}} \sum_{k=1}^{N} \sigma_{k}^{-}|0\rangle^{\otimes N} .
$$

For both states, it is simple to find $X_{A}, Y_{B}$ with $d(A, B)=$ $N / 2$ such that Eq. (1) is not verified. Let us show that they can be prepared by $\mathrm{QCcc}_{2}$. For $|\mathrm{GHZ}\rangle$, we attach one ancilla per site, except for the first one. We define a unitary acting on the $n$th qubit and the $n+1$ ancilla as $u_{n}|0\rangle_{s_{n}} \otimes$ $|0\rangle_{a_{n+1}}=\left|\Phi^{+}\right\rangle_{s_{n}, a_{n+1}} \quad\left(\left|\Phi^{+}\right\rangle_{s_{n}, a_{n+1}}\right.$ : maximally entangled Bell state) as well as $U=\left(\otimes_{n=1}^{N-1} u_{n}\right) \otimes v_{N}$, where $v=$ $\left(\mathbb{1}-i \sigma^{y}\right) / \sqrt{2}$. Applying $U$ to $|\mathbf{0}\rangle_{s, a}$ (which can be done with a QC of depth 2), it generates $\left(\bigotimes_{n=1}^{N-1}\left|\Phi^{+}\right\rangle_{s_{n}, a_{n+1}}\right) \otimes|+\rangle_{s_{N}}$, where $|+\rangle=(|0\rangle+|1\rangle) / \sqrt{2}$. This state can be transformed into $|\mathrm{GHZ}\rangle$ via LOCC. To see this, we apply a local controlled NOT gate between each qubit and its ancilla, yielding $|\Phi\rangle=\sum_{\left\{k_{n}\right\}}\left|k_{1}\right\rangle_{s_{1}}\left(\otimes_{n=2}^{N}\left|k_{n}\right\rangle_{s_{n}} \otimes\left|k_{n-1} \oplus k_{n}\right\rangle_{a_{n}}\right)$, where $k_{n-1} \oplus k_{n}=k_{n-1}+k_{n}(\bmod 2)$, and measure all ancillas in the computational basis. Given the output $\left\{k_{j}\right\}_{j=2}^{N}$, we finally apply $\otimes_{n=2}^{N}\left(\sigma_{n}^{x}\right)^{\sum_{m=2}^{n} k_{m}}$ to the spins. With a similar construction, we can also prove $|\mathbf{0}\rangle \stackrel{\mathrm{QCcc}_{2}}{\longrightarrow}|W\rangle[30]$.

We mention that related constructions for the GHZ state appeared before [48]; see also [49].

Example 2 (fixed points in 1D): In order to show the power of QCcc, we consider the fixed points of the renormalization group procedure introduced in Ref. [50], representing a very general class of states in $1 \mathrm{D}$. To define 
them, we take a chain of $N$ sites with PBC, where each site is associated with three qudits: $C_{n}, L_{n}$, and $R_{n}$ (center, left, and right, respectively). Up to LU transformations, renormalization group fixed points take the form [21]

$$
|\Psi\rangle=\sum_{k=1}^{B} \alpha_{k} \otimes_{n=1}^{N}|k\rangle_{C_{n}}|\psi\rangle_{R_{n}, L_{n+1}},
$$

where $B \in \mathbb{N}, \alpha_{k} \in \mathbb{C}$, while $|\psi\rangle_{R_{n}, L_{n+1}}$ is an entangled state between $R_{n}$ and $L_{n+1}$. Let us show that Eq. (3) can be prepared by $\mathrm{QCcc}_{4}$. We introduce ancillas $C_{n}^{\prime}, L_{n}^{\prime}, R_{n}^{\prime}$ and create maximally entangled states between $R_{n}^{\prime}$ and $L_{n+1}$ with a depth-2 QC. Next, we prepare the qudits $C_{n}$ in the state $\sum_{k} \alpha_{k} \otimes_{n}|k\rangle_{C_{n}}$, which can be done by $\mathrm{QCcc}_{2}$, using ancillas $C_{n}^{\prime}$ and following the steps of Example 1. Using LU, we then prepare the state $|\psi\rangle_{L_{n}^{\prime}, R_{n}}$ between ancillas $L_{n}^{\prime}$ and $R_{n}$, conditioned to the state of $C_{n}$, i.e., $|k\rangle_{C_{n}}|0\rangle_{L_{n}^{\prime}}|0\rangle_{R_{n}} \mapsto|k\rangle_{C_{n}}|\psi\rangle_{L_{n}^{\prime}, R_{n}}$. Finally, we use the entangled pairs between $R_{n}^{\prime}$ and $L_{n+1}$ to teleport $L_{n}^{\prime}$ to $L_{n+1}$, which can be done via LOCC [26].

Example 3 (the toric code): Finally, let us consider qubits in a 2D lattice with $\operatorname{PBC}\left(M=N^{2}\right)$, where $i \in \Lambda$ has two coordinates, $i=\left(i_{1}, i_{2}\right)$, and focus on the toriccode state $|\mathrm{TC}\rangle[16]$. For $N$ even, the TC can be defined by placing the qubits at the vertices of a square lattice. Let $P$ be the set of all plaquettes composed of four contiguous vertices forming a square. We divide them into two types, $P_{A}$ and $P_{B}$, following a chessboard pattern. For each A plaquette $p \in P_{A}$, we introduce $X_{p}=\bigotimes_{i \in p} \sigma_{i}^{x}$, and define $|\mathrm{TC}\rangle \propto$ $\prod_{p \in P_{A}}\left(\mathbb{1}+X_{p}\right)|0\rangle^{\otimes M}$. We also set $S_{j}^{\alpha}=\bigotimes_{k=1}^{N} \sigma_{j, k}^{\alpha}$ for $j=1, \ldots, N$. It is well known that the TC can not be prepared by $\mathrm{QC}_{\ell}$ for $\ell$ independent of $N$ [51]. This can also be seen by noticing that Eq. (1) is not satisfied choosing $X_{A}=S_{1}^{x}, Y_{B}=S_{N / 2+1}^{x}$. Let us show $|\mathbf{0}\rangle \stackrel{\mathrm{QCcc}_{16}}{\longrightarrow}|\mathrm{TC}\rangle$. We do this following [52] (see also [53]). For each $p \in P_{A}$, we include an ancilla, $a_{p}$, in the vertex at the upper-left corner of $p$. Next, we define the unitary $V=\prod_{p \in P_{A}} V_{p}$, with $V_{p}=\frac{1}{2}\left[\left(\mathbb{1}+X_{p}\right) \otimes \mathbb{1}_{a_{p}}+\left(\mathbb{1}-X_{p}\right) \otimes \sigma_{a_{p}}^{x}\right] . V_{p}$ may be implemented using 8 nearest-neighbor gates: (i) we introduce 4 additional ancillas at the upper-left corner of $p$, denoted by $Q$; (ii) we swap them with the qubits at the vertices of $p$ (with 4 gates); (iii) we apply (locally) $V_{p}$ to the five ancillas in $Q$; (iv) we swap back the qubits in $Q$ with the vertices of $p$. Then, dividing $P_{A}$ into two subsets, $P_{A}^{\prime}, P_{A}^{\prime \prime}$, such that all plaquettes in each subset share no common qubit, we can implement $V$ by acting in parallel on all $p \in P_{A}^{\prime}$, then on all in $p \in P_{A}^{\prime \prime}$, resulting in a QC of depth 16 . After applying $V$, we measure $\sigma^{z}$ in all the ancillas $a_{p}$ with outcomes $k_{p}= \pm 1$. The fact that $\prod_{p \in P_{A}} X_{p}=\mathbb{1}$ implies that the product of all $k_{p}$ equals 1 [54]. The resulting state is

$$
\left|\psi_{k}\right\rangle \propto \prod_{p \in P_{A}}\left(\mathbb{1}+k_{p} X_{p}\right)|0\rangle^{\otimes M} .
$$

Finally, it is easy to see that given a set of $k_{p}= \pm 1$ whose product equals 1 , it is always possible to find $Z_{k}$, a product of $\sigma^{z}$ operators, such that $Z_{k}\left(\mathbb{1}+k_{p} X_{p}\right) Z_{k}=$ $\left(\mathbb{1}+X_{p}\right), \forall p$. Thus, by applying the $\mathrm{LU} Z_{k}$ we recover the TC deterministically.

In summary, LOCC enlarges the set of states that can be prepared deterministically. One could wonder whether all states may be realized in this way. This is not the case, and only states satisfying an entanglement area law, similar to that characterizing ground states of local Hamiltonians [55], may be prepared. To see this, we have to consider a sequence of states $\left\{|\psi\rangle_{M}\right\}_{M}$ on lattices of increasing size. We assume that $|\psi\rangle_{M}$ is prepared by $\mathrm{QCcc}_{\ell}$, where $\ell$ is independent of $M$, and denote by $S_{0}^{\psi /}\left(A: A^{c}\right)$ the max entropy between $A \subset V$ and its complement $A^{c}=V / A$, which upper bounds the von Neumann entropy [24]. We also call $\partial A$ the boundary of $A$ and denote by $|A|$ the number of qudits in $A$.

Definition 3 (entanglement area law): A sequence of states $\left\{\left|\psi_{M}\right\rangle\right\}_{M}$ obeys an entanglement area law if for all $A \subset V, S_{0}^{\psi_{M}}\left(A: A^{c}\right) \leq c|\partial A|$, where $c$ is a constant independent of $M$.

Proposition 2: Any sequence of states $\left\{\left|\psi_{M}\right\rangle\right\}_{M}$ prepared by $\mathrm{QCcc}_{\ell}$ (with $\ell$ independent of $M$ ) satisfies an entanglement area law. See [30] for a proof.

Phases of matter.-QCs appear naturally in the standard classification of topological phases of matter [2-6]. Colloquially, for ground states of gapped, local Hamiltonians, it is known that if two states are in the same phase (i.e., their parent Hamiltonians are connected by a differentiable path of gapped, local Hamiltonians), then they are mapped onto one another by a "low-depth" QC. Inverting the logic, one could use QCs to define equivalence classes. However, some care must be taken: indeed if $\left|\psi_{2}\right\rangle=U\left|\psi_{1}\right\rangle$, and $\left|\psi_{3}\right\rangle=V\left|\psi_{2}\right\rangle$ with $U, V \in \mathcal{Q} C_{\ell}$, then to transform $\left|\psi_{1}\right\rangle$ to $\left|\psi_{3}\right\rangle$ may require an operation in $\mathcal{Q C}_{2 \ell}$, meaning that one has to allow for the depth to change. One way to do this is to define an equivalence relation between state sequences, $\Psi=\left\{\left|\psi_{M}\right\rangle \in H_{M}\right\}_{M=M_{0}}^{\infty}$, for lattices of increasing size, where $M_{0} \in \mathbb{N}$ : one can say that $\Psi \sim \Phi$ if $\exists U_{M} \in \mathcal{Q} \mathcal{C}_{f(M)}$ such that ||$\left|\psi_{M}\right\rangle-U_{M}\left|\varphi_{M}\right\rangle|| \stackrel{M \rightarrow \infty}{\longrightarrow} 0$. Here, $f(M)$ is a function that grows sufficiently slowly in $M$. For example, ground states of gapped, local Hamiltonians in the same phase are equivalent by this definition choosing $f(M)$ to be a polylogarithmic function of $M[56,57]$ (where one also allows for a number of ancillas polylogarithmic in $M$ ); see also Refs. [58-61].

We wish to extend this definition by replacing QCs with QCcc (and without restricting to ground states). To do that, we allow for approximate preparation protocols, where a pure state may be mapped onto a mixed state $\rho$, as we now explain [62]. A given preparation protocol in $\mathrm{QCcc}_{\ell}$ (where ancillas are traced out at the end) defines a quantum channel $\mathcal{C}$ [24]. If a pure initial state $|\varphi\rangle$ can be mapped 
onto the (mixed) state $\sigma$ for some $\mathcal{C}$ defined in this way, we will write $|\varphi\rangle \stackrel{\mathrm{QCcc}_{\ell}}{\rightarrow} \sigma$. We will also use the symbol $\mathrm{QCcc}_{\ell}^{(k)}$ to denote transformations obtained by composing $k$ channels defined by preparation protocols in $\mathrm{QC}_{\mathrm{cc}_{\ell}}\left\{\mathcal{C}_{j}\right\}_{j=1}^{k}$. Then, we may define an equivalence relation as follows. First, given two sequences, $\Psi, \Phi$, we write $\Psi \mapsto \Phi$ if $\exists k \in \mathbb{N}$ and a sequence of (mixed) states $\left\{\sigma_{M}\right\}_{M=M_{0}}^{\infty}$, such that $\left|\psi_{M}\right\rangle \stackrel{\mathrm{QCcc}_{f(M)}^{(k)}}{\longrightarrow} \sigma_{M}$ and $\| \sigma_{M}-\left|\varphi_{M}\right\rangle\left\langle\varphi_{M}\right| \|_{1} \stackrel{M \rightarrow \infty}{\longrightarrow} 0$, where $\|\cdot\|_{1}$ is the trace norm. Here, analogously to the case of QCs, we choose $f(M)$ to be a polylogarithmic function of $M$. Finally, we say that $\Psi$ is QCcc-equivalent to $\Phi$ if $\Psi \mapsto \Phi$ and $\Phi \mapsto \Psi$. Note that this more complicated definition is needed to ensure symmetry and transitivity (which simply follows from the contractivity of the trace norm).

Solving the full classification problem is expected to be very hard. However, based on Example 2, we can give a strong result in $1 \mathrm{D}$, proving that all translational invariant MPS with fixed bond dimension belong to the trivial class [30].

Theorem 1: In 1D, all translational invariant MPS with fixed bond dimension are in the same phase as the trivial state.

A proof of this theorem is given in [30]. QCcc classes are strictly larger than those in the standard classification of topological phases, as exemplified by the TC. In fact, it is natural to conjecture that the same is true for all nonchiral topologically ordered states, although this problem goes beyond the scope of this work. The suggested classification is expected to have practical ramifications in preparation protocols with noisy intermediate-scale quantum devices, as states in the trivial phase may be prepared efficiently with operations already at hand: geometrically local gates and on-site measurements. At the same time, from the fundamental standpoint, QCcc provide a unified framework where the locality-based classification of states in QMBP and QIT meet.

Unitary operations.-It is known that allowing for postselection processes the power of quantum computers increases [63,64]. Postselection, however, has practical limitations due to the large number of times that a computation must be performed. Here, we take a different point of view and ask whether, combining LOCC and QC, one can implement "deterministically" a larger set of unitary operations beyond QCs [25]. This is different from the state-transformation protocols since now we want unitary actions on all possible input states.

Let us now introduce a general scheme to implement unitary operators that involves QCs and LOCC [65]. First, we prepare a state $|\phi\rangle_{a}$ on the ancillas, using only QCs and LOCC as in the state-transformation protocol discussed before. Then, given an input state $|\psi\rangle$, the procedure consists of applying a depth- $\ell$ quantum circuit
$V$ (including ancillas and LU) to the pair system ancilla, followed by LOCC. In particular, we consider operations

$$
|\psi\rangle \vec{U}_{s}^{\alpha}\left(\otimes_{k}\left\langle\alpha_{k}\right|\right) V_{s a}\left(|\psi\rangle_{s} \otimes|\phi\rangle_{a}\right) .
$$

The subscripts $s$ and $a$ label system and ancilla, $\left|\alpha_{k}\right\rangle$ is an element of a local orthonormal basis for the ancillas, while $U_{s}^{\alpha} \in \mathcal{L U}$, which might depend on the outcomes $\alpha_{k}$. We are interested in the special cases where the action [Eq. (5)] defines a unitary operation.

Definition 4 (LOCC-assisted quantum circuits): $\mathcal{Q} C \operatorname{cc}_{\ell} \subset \mathcal{U}$ is the set unitary operators that can be implemented (deterministically) by a QC of depth $\ell$ with the help of ancillas using the protocol [Eq. (5)].

Note that in the above we also require $|\mathbf{0}\rangle \stackrel{\mathrm{QCcc}_{\ell}}{\rightarrow}|\phi\rangle$.

Trivially, $\mathcal{Q C}_{\ell} \subseteq \mathcal{Q} \mathcal{C c c}_{\ell}$. In fact, the inclusion is strict, as we illustrate with a specific construction that, on the one hand, ensures that the map [Eq. (5)] is unitary, while, on the other, allows us to implement operators beyond QCs. Before that, we need to recall two notions in QIT. The first one is that of Clifford operators [66,67]. To define them, we introduce the set $\mathcal{Q}$ of tensor products of Pauli operators, i.e., $\mathcal{Q}=\left\{\otimes_{i=1}^{M} \sigma_{i}^{\alpha_{i}}, \alpha_{i}=0, x, y, z\right\}$, where $\sigma_{j}^{0}=\mathbb{1}_{j}$. Then, $U \in \mathcal{U}$ is a Clifford operator if for any $s \in \mathcal{Q}, U^{\dagger} s U=s^{\prime} \in \mathcal{Q}$ (possibly up to a factor). The second one is that of locally maximally entangled states [68]. They are defined as the states $|\varphi\rangle_{s}$ for which there exist LU that create a maximally entangled state between the spins and the ancillas, i.e., there exist $u_{i} \in \mathrm{LU}$ such that $|R\rangle=\bigotimes_{i=1}^{M} u_{i}\left(|\varphi\rangle_{s} \otimes|\mathbf{0}\rangle_{a}\right)$ fulfills $\operatorname{tr}_{a}(|R\rangle\langle R|)=\mathbb{1}_{s}$.

Now, our construction is as follows. First, we append one ancilla per site, $a_{n}$, and prepare $|\phi\rangle_{a}$ by $\mathrm{QCcc}_{\ell}$. We require that $|\phi\rangle$ is a stabilizer state, i.e., the unique common eigenstate of a set of commuting elements of $\mathcal{Q}$. This implies that $|\phi\rangle$ is also locally maximally entangled [68]. Thus, adding one additional ancilla per site, $a_{n}^{\prime}$, there exists $V \in \mathcal{L U}$ such that $|R\rangle_{a, a^{\prime}}=V|\phi\rangle_{a}|0\rangle_{a^{\prime}}$ is maximally entangled. This implies that the map $|\psi\rangle_{s} \mapsto$ $d^{M}{ }_{s, a^{\prime}}\left\langle\Phi^{+} \mid R\right\rangle_{a, a^{\prime}} \otimes|\psi\rangle_{s}$ equals the action of a unitary operator $U$ [69] (where $\left|\Phi^{+}\right\rangle_{s, a}$ is the maximally entangled Bell state between system $s$ and ancillas $a$ ). Furthermore, since $|\phi\rangle$ is a stabilizer, one can choose $V$ such that $U$ is a Clifford operator [30]. Then, for any input $|\psi\rangle, U|\psi\rangle$ can be implemented deterministically using LOCC. To do this, we perform a Bell measurement on the qubits $s_{n}$ and $a_{n}^{\prime}$. This produces $U\left(\otimes_{n} \sigma^{\alpha_{n}}\right)|\psi\rangle_{a}$, where $\alpha_{n}$ depend on the values of the measurement. Since $U$ is a Clifford operator, $U\left(\otimes_{n} \sigma^{\alpha_{n}}\right)=w U$, with $w \in \mathcal{Q}$ and hence $w \in \mathcal{L U}$, so that $U|\psi\rangle$ is recovered applying $w^{\dagger} \in \mathcal{L U}$.

Example 4 (the GHZ and TC unitaries): Consider the GHZ state. It is a stabilizer state prepared by $\mathrm{QCcc}_{2}$, and thus it may be used to implement a unitary operator. To see this explicitly, starting from $|\mathrm{GHZ}\rangle_{s}$, we first apply a single phase gate to one of the state qubits. Then, we prepare a 
maximally entangled state with an ancillary system where all ancillas are initialized in $|+\rangle$ by simultaneously applying controlled NOT gates to each system-ancilla pair (with the system being the target), thus obtaining a state $|R\rangle_{s, a}$. It is easy to see that the action $|\psi\rangle_{a^{\prime}} \mapsto d^{M}{ }_{a, a^{\prime}}\left\langle\Phi^{+} \mid R\right\rangle_{s, a} \otimes$ $|\psi\rangle_{a^{\prime}}$ corresponds to the unitary $U_{\mathrm{GHZ}}=\left(\mathbb{1}+i \sigma_{x}^{\otimes M}\right) / \sqrt{2}$. Importantly, $U_{\mathrm{GHZ}}$ is a Clifford operator and thus may be implemented by LOCC. Also, $U_{\mathrm{GHZ}} \notin \mathcal{Q C}_{\ell}$ for $\ell<N$ because $U^{\dagger} \sigma_{1}^{z} U$ is a string of Pauli matrices over the whole system. Similarly, starting from the TC, we can construct a unitary $U_{\mathrm{TC}} \in \mathcal{Q} C \mathrm{cc}_{16}$ such that $U_{\mathrm{TC}}|\mathbf{0}\rangle$ is locally equivalent to $|\mathrm{TC}\rangle$ [30], implying that $U_{\mathrm{TC}} \notin \mathcal{Q \mathcal { C } _ { \ell }}$ for $\ell<N / 4$.

Outlook. - In this Letter, we have introduced a paradigm to classify states and operations based on a notion of locality inspired by both QIT and QMBP, arguing for its fundamental relevance and potential practical importance. Our work raises several questions. First, we have seen examples of topologically ordered states in the trivial class, but an obvious question is whether all representatives of nontrivial phases may be prepared by QCcc. A similar problem holds for chiral states, which we have not addressed. Moreover, we have considered here QCs composed of local gates; it is natural to wonder how our conclusions are modified using nonlocal gates instead. Finally, ideas related to those presented here may lead to a classification for unitary operators: although this requires getting around some subtleties, we expect that such a classification will be different from the one for the corresponding Choi-Jamiolkowski states [24]. We leave these questions for future work.

We thank Alex Turzillo for very useful discussions. We acknowledge support by the EU Horizon 2020 program through the ERC Advanced Grant QUENOCOBA No. 742102 and by the DFG (German Research Foundation) under Germany's Excellence StrategyEXC-2111-390814868. This project has received funding from the European Unions Horizon 2020 research and innovation program under grant agreement No. 899354.

[1] R. Horodecki, P. Horodecki, M. Horodecki, and K. Horodecki, Rev. Mod. Phys. 81, 865 (2009).

[2] X. Chen, Z.-C. Gu, and X.-G. Wen, Phys. Rev. B 82, 155138 (2010).

[3] M. B. Hastings, Phys. Rev. B 88, 165114 (2013).

[4] B. Zeng, X. Chen, D.-L. Zhou, and X.-G. Wen, arXiv: 1508.02595 .

[5] B. Zeng and X.-G. Wen, Phys. Rev. B 91, 125121 (2015).

[6] C.-K. Chiu, J. C. Y. Teo, A. P. Schnyder, and S. Ryu, Rev. Mod. Phys. 88, 035005 (2016).

[7] Y. Li, X. Chen, and M. P. A. Fisher, Phys. Rev. B 98, 205136 (2018).

[8] B. Skinner, J. Ruhman, and A. Nahum, Phys. Rev. X 9, 031009 (2019).
[9] R. Fan, S. Vijay, A. Vishwanath, and Y.-Z. You, Phys. Rev. B 103, 174309 (2021).

[10] C.-M. Jian, Y.-Z. You, R. Vasseur, and A. W. W. Ludwig, Phys. Rev. B 101, 104302 (2020).

[11] S. Choi, Y. Bao, X.-L. Qi, and E. Altman, Phys. Rev. Lett. 125, 030505 (2020).

[12] M. J. Gullans and D. A. Huse, Phys. Rev. X 10, 041020 (2020).

[13] M. Ippoliti, M. J. Gullans, S. Gopalakrishnan, D. A. Huse, and V. Khemani, Phys. Rev. X 11, 011030 (2021).

[14] J. Preskill, Quantum 2, 79 (2018).

[15] K. Satzinger, Y. Liu, A. Smith, C. Knapp, M. Newman, C. Jones, Z. Chen, C. Quintana, X. Mi, A. Dunsworth et al., arXiv:2104.01180.

[16] A. Y. Kitaev, Ann. Phys. (Amsterdam) 303, 2 (2003).

[17] D. M. Greenberger, M. A. Horne, and A. Zeilinger, in Bells Theorem, Quantum Theory, and Conceptions of the Universe, edited by M. Kafatos, Fundamental Theories of Physics Vol. 37 (Springer, Dordrecht, 1989), https://doi.org/ 10.1007/978-94-017-0849-4_10.

[18] W. Dür, G. Vidal, and J. I. Cirac, Phys. Rev. A 62, 062314 (2000).

[19] M. Fannes, B. Nachtergaele, and R. F. Werner, Commun. Math. Phys. 144, 443 (1992).

[20] D. Perez-Garcia, F. Verstraete, M. Wolf, and J. Cirac, Quantum Inf. Comput. 7, 401 (2007).

[21] J. I. Cirac, D. Perez-Garcia, N. Schuch, and F. Verstraete, Ann. Phys. (Amsterdam) 378, 100 (2017).

[22] N. Schuch, D. Pérez-García, and I. Cirac, Phys. Rev. B 84, 165139 (2011).

[23] Often, gates are restricted to belong to some finite (universal) gate set. Here, since we focus on properties related to locality, we instead consider as a valid gate any two-qudit unitary operator acting on nearest neighbors.

[24] M. A. Nielsen and I. Chuang, Quantum Computation and Quantum Information (Cambridge University Press, Cambridge, England, 2002).

[25] D. Gottesman and I. L. Chuang, Nature (London) 402, 390 (1999).

[26] C. H. Bennett, G. Brassard, C. Crépeau, R. Jozsa, A. Peres, and W. K. Wootters, Phys. Rev. Lett. 70, 1895 (1993).

[27] R. Raussendorf and H. J. Briegel, Phys. Rev. Lett. 86, 5188 (2001).

[28] F. Verstraete and J. I. Cirac, Phys. Rev. A 70, 060302(R) (2004).

[29] C. H. Bennett, D. P. DiVincenzo, J. A. Smolin, and W. K. Wootters, Phys. Rev. A 54, 3824 (1996).

[30] See Supplemental Material at http://link.aps.org/supplemental/ 10.1103/PhysRevLett.127.220503 which includes Refs. [3147], for a detailed proof of the statements presented in the main text.

[31] P. Arrighi, Nat. Comput. 18, 885 (2019).

[32] T. Farrelly, Quantum 4, 368 (2020).

[33] P. Arrighi, V. Nesme, and R. Werner, J. Comput. Syst. Sci. 77, 372 (2011).

[34] T. C. Farrelly and A. J. Short, Phys. Rev. A 89, 012302 (2014).

[35] D. Gross, V. Nesme, H. Vogts, and R. F. Werner, Commun. Math. Phys. 310, 419 (2012). 
[36] J. I. Cirac, D. Perez-Garcia, N. Schuch, and F. Verstraete, J. Stat. Mech. (2017) 083105.

[37] M. B. Şahinoğlu, S. K. Shukla, F. Bi, and X. Chen, Phys. Rev. B 98, 245122 (2018).

[38] B. R. Duschatko, P. T. Dumitrescu, and A. C. Potter, Phys. Rev. B 98, 054309 (2018).

[39] Z. Gong, L. Piroli, and J. I. Cirac, Phys. Rev. Lett. 126, 160601 (2021).

[40] L. Fidkowski, H. C. Po, A. C. Potter, and A. Vishwanath, Phys. Rev. B 99, 085115 (2019).

[41] L. Piroli, A. Turzillo, S. K. Shukla, and J. I. Cirac, J. Stat. Mech. (2021) 013107.

[42] L. Piroli and J. I. Cirac, Phys. Rev. Lett. 125, 190402 (2020).

[43] M. A. Nielsen, Phys. Rev. Lett. 83, 436 (1999).

[44] W. van Dam and P. Hayden, arXiv:quant-ph/0204093.

[45] R. Bhatia, Matrix Analysis (Springer Science \& Business Media, New York, 2013), Vol. 169.

[46] D. Schlingemann, arXiv:quant-ph/0111080.

[47] M. Van den Nest, J. Dehaene, and B. De Moor, Phys. Rev. A 69, 022316 (2004).

[48] C. Meignant, D. Markham, and F. Grosshans, Phys. Rev. A 100, 052333 (2019).

[49] A. B. Watts, R. Kothari, L. Schaeffer, and A. Tal, in Proceedings of the 51st Annual ACM SIGACT Symposium on Theory of Computing (Association for Computing Machinery, New York, 2019), pp. 515-526, https://doi .org/10.1145/3313276.3316404.

[50] F. Verstraete, J. I. Cirac, J. I. Latorre, E. Rico, and M. M. Wolf, Phys. Rev. Lett. 94, 140601 (2005).

[51] S. Bravyi, M. B. Hastings, and F. Verstraete, Phys. Rev. Lett. 97, 050401 (2006).
[52] R. Raussendorf, S. Bravyi, and J. Harrington, Phys. Rev. A 71, 062313 (2005).

[53] M. Aguado, G. K. Brennen, F. Verstraete, and J. I. Cirac, Phys. Rev. Lett. 101, 260501 (2008).

[54] Indeed, if $\prod_{p \in P_{A}} k_{p}=-1$, using $\prod_{p \in P_{A}} X_{p}=\mathbb{1}$ one can easily show that $\left\langle\psi_{k} \mid \psi_{k}\right\rangle=0$, where $\left|\psi_{k}\right\rangle$ is defined in (4).

[55] J. Eisert, M. Cramer, and M. B. Plenio, Rev. Mod. Phys. 82, 277 (2010).

[56] A. Coser and D. Pérez-García, Quantum 3, 174 (2019).

[57] J. Haah, M. B. Hastings, R. Kothari, and G. H. Low, SIAM J. Comput. SPECIAL SECTION FOCS18 (2021).

[58] T. J. Osborne, Phys. Rev. Lett. 97, 157202 (2006).

[59] T. J. Osborne, Phys. Rev. A 75, 032321 (2007).

[60] S. Bachmann, S. Michalakis, B. Nachtergaele, and R. Sims, Commun. Math. Phys. 309, 835 (2012).

[61] Y. Huang and X. Chen, Phys. Rev. B 91, 195143 (2015).

[62] We note that other definitions that do not modify our conclusions are possible.

[63] S. Aaronson, Proc. R. Soc. A 461, 3473 (2005).

[64] N. Schuch, M. M. Wolf, F. Verstraete, and J. I. Cirac, Phys. Rev. Lett. 98, 140506 (2007).

[65] Note that, even without measurements, i.e., by simply allowing for additional ancillas, the set of unitary operations is enlarged [30].

[66] D. Gottesman, Ph.D. thesis, Caltech, 1997.

[67] D. Gottesman, Phys. Rev. A 57, 127 (1998).

[68] C. Kruszynska and B. Kraus, Phys. Rev. A 79, 052304 (2009).

[69] M. M. Wolf, Lecture notes available at http://www-m5.ma .tum.de/foswiki/pubM, 5 (2012). 